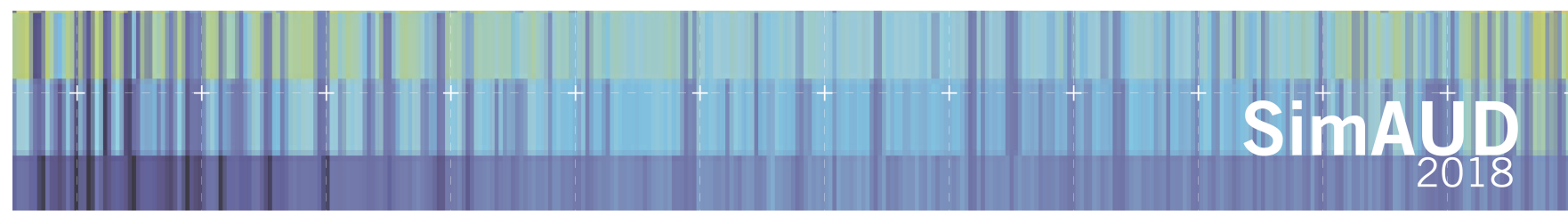

\title{
Artificial Neural Network-Based Modelling for Daylight
}

\section{Evaluations}

\author{
Clara-Larissa Lorenz ${ }^{1}$, Michael Packianather ${ }^{2}$, A. Benjamin Spaeth ${ }^{3}$ and Clarice Bleil De Souza ${ }^{4}$ \\ ${ }^{1}$ Cardiff University \\ Cardiff, U.K. \\ ${ }^{2}$ Cardiff University \\ Cardiff, U.K. \\ Lorenzc4@cardiff.ac. packanatherms@cardiff \\ uk \\ .ac.uk

\section{${ }^{3}$ Cardiff University} \\ Cardiff, U.K. \\ spaetha@cardiff.ac.uk \\ ${ }^{4}$ Cardiff University \\ Cardiff, U.K. \\ bleildesouzac@cardiff \\ .ac.uk
}

\begin{abstract}
The climate based Daylight Autonomy (DA) metric has been gaining ground in the field of sustainable building design as a measure for the amount of daylight within spaces and associated energy savings. In this study, Artificial Neural Networks (ANNs) were used to predict DA levels in interior spaces as an alternative to computationally expensive simulations. Research was carried out in three phases of increasing complexity: First, a neural network was trained and validated for a single design space. Subsequently, the window design was altered and a neural network was trained and tested on its ability to predict DA levels according to changes in window design. Lastly, the neural network was trained to account for the effects of shading from an external obstruction. After sufficient training, the ANN, during the recall stage, was able to predict DA, on average, within 3 DA short of the simulated DA results for both the shaded and unobstructed scenario. The results obtained show the potential of neural networks as a prediction tool for estimating Daylight Autonomy.
\end{abstract}

\section{Author Keywords}

Artificial neural network; climate-based daylight modeling; daylight autonomy, daylight performance; machine learning

\section{ACM Classification Keywords \\ I.6.1 SIMULATION AND MODELING; I.2.6 \\ CONNECTIONISM AND NEURAL NETS}

\section{INTRODUCTION}

Daylight is an essential physiological and psychological human need that has been shown to improve human wellbeing and work performance [1, 2]. As a measure for daylight, current building standards and guidelines (e.g. BS 8206-2:2008; BR209-2011) still predominantly rely on the Daylight Factor (DF). This metric however has been criticized for its inability to distinguish between a better or worse design approach as it takes into account neither climate, orientation nor shading [3]. In place, climate-based

daylight metrics have been recommended as a design driver to evaluate design solutions.

This paper employs the climate based Daylight Autonomy metric (DA) and investigates the potential of applying artificial neural networks (ANNs) to predict this metric. By proving the potential of ANNs to learn or mimic the behaviour of simulation tools, it aims to establish a basis for ANNs to partially replace time-consuming simulations in design optimization processes.

The next part of the paper gives the definition for DA, describes the basic construct of ANNs and explores the current application of ANNs as prediction tools. In the third section, the four methodological experiments undertaken in this study are described, after which the results are reported in section 4. The last section evaluates the findings and draws the conclusions for this paper.

\section{ARTIFICIAL NEURAL NETWORK PREDICTIONS OF DAYLIGHT AUTONOMY}

Daylight Autonomy (DA) measures the amount of occupied hours in a year, in which a given illuminance target can be met by daylight alone [4]. For office work, 300 lux is a typical target taken as the illuminance threshold. To illustrate, a DA of $80 \%$ reports that artificial lighting may not be required for $80 \%$ of occupied hours in a year. This metric and variations of the metric (such as Spatial Daylight Autonomy and Useful Daylight Illuminances) are thus commonly used in lighting design for designing daylight harvesting systems [5]. Time-consuming climate based simulations at hourly or smaller time steps are needed to calculate the annual result of this metric. As such, research has also ventured into the field of machine learning and proposed neural networks to tackle the problem of computational expensive simulations while maintaining prediction accuracy [6].

Artificial neural networks are the mathematical description of 'brain-like' systems that have been developed with biological neural networks as their core concept [7]. Characteristically, ANNs are a construct arranged in an 
input layer, one or more hidden layers in the middle and an output layer (Figure 1). The layers consist of a variable number of neurons with variable connection strengths. The connection strengths between the neurons of each layer are established by providing the ANN with a set of training data and the respective results, to which it then develops a pattern of connections that replicate complex functions and mimic non-linear behaviour. Depending on the applied learning rules and the transfer functions between neurons, the networks can be trained as prediction models to compute continuous output such as illuminances (function approximation)[8], or binary output that classifies data into categories (classification), e.g. illuminance categories such as Useful Daylight Illuminances [9].

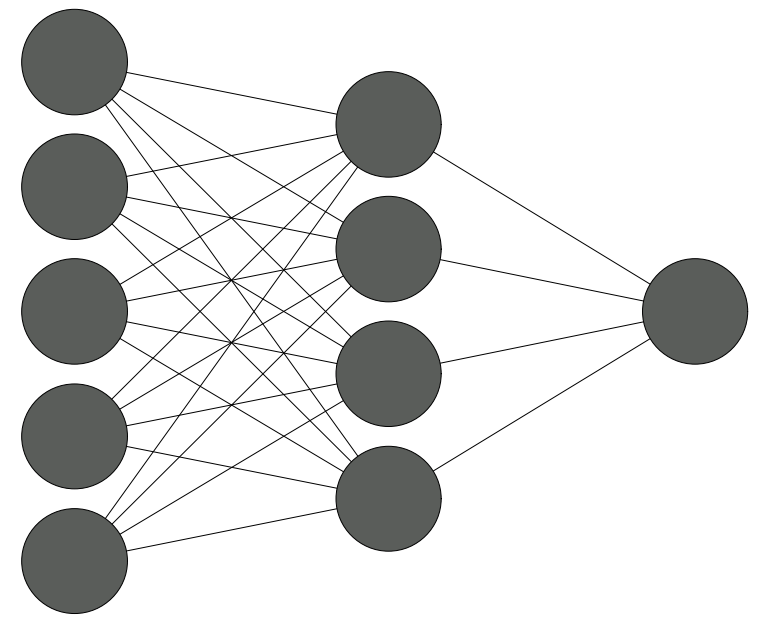

Input Layer

Hidden Layer

Output Layer

Figure 1. Neural network architecture with five input neurons, four neurons in the hidden layer and one neuron in the output layer.

One of the studies that applied ANN-based modelling successfully predicted illuminance levels in an office building with a percentage error of $3 \%$ compared to field measurements [6]. The study used Illuminance measurements alongside time variables and weather data to train the network. In another study, the illuminance results obtained from a trained neural network were used to optimize the slat angle of blinds [10]. Good prediction results were also obtained in a study that applied neural networks to estimate sky luminances. The study showed an overall better performance of the ANN over CIE sky models [11].

In an effort to evaluate the daylight performance of a building, a more recent study used ANNs for hourly predictions of the climate-based metric UDI (Useful Daylight Illuminances). The network was able to correctly classify the UDI category for most days of the year and showed a better performance than support vector machines, another machine learning technique [9]. ANNs also yielded promising results for annual predictions of the Daylight Autonomy metric [12]. Although the studies show the ANNs potential as an alternative computational model for daylight predictions, one of the major drawbacks stems from the effort required to collect or generate the data required for ANN training. Additionally, Neural Network models remain as a black box [13] and the sensitivity of prediction results towards a selected neural network architecture as well as the randomly selected initial weight settings make their robustness questionable.

This research models artificial neural networks to predict annual DA levels for changes in façade design, specifically window dimension and window location. The data is generated from daylight simulations. So as to improve reliability and robustness of the networks, the study ran multiple trials and optimized the neural network architecture for each training set individually before applying the ANN to predict the DA metric.

\section{METHODOLOGY}

\subsection{ANN Settings}

A neural network model was developed by providing a set of input output pairs to the network to undergo supervised training. In this case, the design variables were the input and their corresponding DA values the output. This study employed a feed-forward neural network that uses the backpropagation training. Back-propagation neural networks are popular for function approximation problems [14] and use gradient descent to adjust connection weights between neurons. As such, there is risk of getting stuck in local minima. To mitigate this risk, network settings such as the speed or learning rate and the step size in which the network weights are adjusted as well as the initial weight setting itself become crucial factors affecting the outcome of ANN training [15]. Therefore, the Levenberg-Marquardt algorithm was implemented in conjunction with backpropagation for a faster convergence during training and increased robustness [16], as it switches between gradientdescent and a Newton-like update of the weights. The neural networks were modeled and trained in MATLAB and each network was trained ten times with randomised initial weight settings.

As the connections between neurons are at the basis of neural network modeling, the ANN architecture, specifically the number of neurons in the hidden layer, are a paramount influencer of ANN models [17]. A typical approach to determining the optimum number of hidden neurons is one of trial and error. Research has recommended a pyramidal structure in which the number of neurons is smaller than the number of input neurons and bigger than then the number of output neurons [18]. Similar studies in the field however found predictions results to be more accurate with a larger number of neurons in the hidden layers [19]. For this study, a script was written that tests the performance of ANN models starting from a small number of three hidden neurons up to 25 hidden neurons.

All input data was normalised to the range of -1 and 1 before training and a tan-sigmoidal activation function was 
employed in between the input and hidden layer and the hidden and output layer. To mitigate overfitting [20], the training data set was subdivided into a validation set of $30 \%$ and the default MATLAB setting of six maximum validation failures was selected to initiate an early stopping of neural network training before completion of 1000 training epochs.

\subsection{Simulation Design}

A generic model of $10 \times 10 \mathrm{~m}$ with and a cantered window and a window head height of $2.7 \mathrm{~m}$ was chosen as the base model for daylight evaluations (Figure 2). The daylight simulations were run on Diva for Rhino, a validated and Radiance-based simulation tool [21]. DA was calculated for 121 sensor points at a work plane height of $0.8 \mathrm{~m}$.

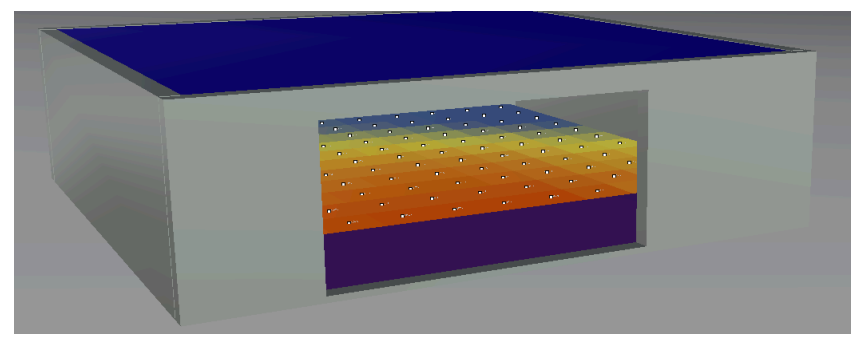

Figure 2. Generic model used for daylight simulations.

Window length and location of the window on the south facing façade were varied by $1 \mathrm{~m}$ in each alteration with resulting designs shown in Figure 4-7.

In an additional scenario, a one-storey building was placed diagonally across the model in $8 \mathrm{~m}$ distance from it. The training data generated from this scenario was used to investigate the ability of the ANN model to cope with additional noise in the training data.

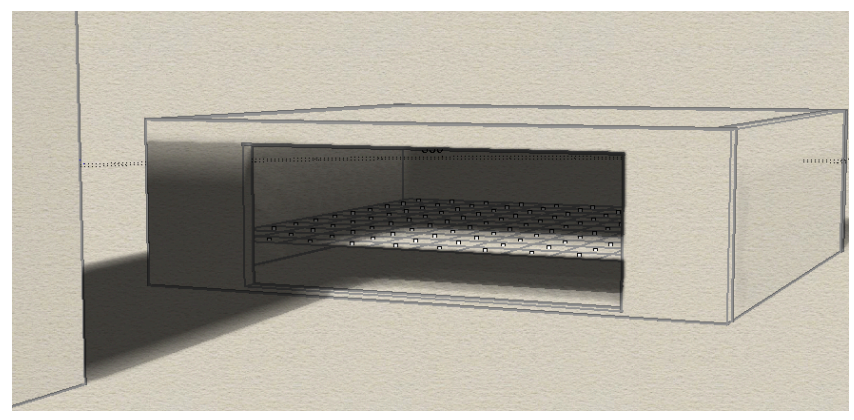

Figure 3. Overshadowing from an external obstruction.
The training and testing of ANN models was undertaken in the four parts:

\section{Neural Network Validation}

Daylight simulation results for all 121 sensor points of design A (Figure 4) were extracted and attributed a sensor point ID according to the distance from each sensor point to the center of the window. The data was then used to generate the ANN input and target data. $90 \%$ of the data was used for training and early stopping; the remaining $10 \%$ of the data was withheld for testing ANN prediction accuracy. This was done once with the data that was generated from the unshaded scenario and repeated a second time with the data generated from the model that received shading from a neighboring building.

\section{Predicting DA for Variations in Window Dimension}

Simulations results were extracted from A to I (Figure 4) and window width was added as an input feature for neural network training. In a first run, the input and target data for $\mathrm{B}$ was withheld from training. After training and optimizing the ANN architecture, DA levels were predicted for all sensor points in B. In a second run, the ANN was retrained using the input and target data from $A$ to $G$ and $I$ as training data and the data for $\mathrm{H}$ was withheld for testing. After training and optimizing the ANN architecture, DA levels were predicted for all sensor points in $\mathrm{H}$. This was done for both the unshaded and shaded scenario.

\section{Predicting DA for Variations in Window Location}

Input and target data was extracted for simulations $\mathrm{H} 1$ to H7 (Figure 6). The location of the centre point of the window was added as an input feature for training the ANN. In a first run, data for H3 was withheld for testing, then another network was trained without the data set for H5. The optimised and trained networks were used to predict DA levels for $\mathrm{H} 3$ and $\mathrm{H} 5$ and prediction results are compared in the next section. Again this was done for both the obstructed and unobstructed scenario.

\section{Predicting DA for Variations in Window Dimension and Location}

In a final test, all data points for window dimension and location (Figures 4, 5 and 7) with the exception of data for $\mathrm{G}$ was used to train another network. After training and optimization of the network architecture, the network was used to predict DA levels for G1, G2, G6 and G7.
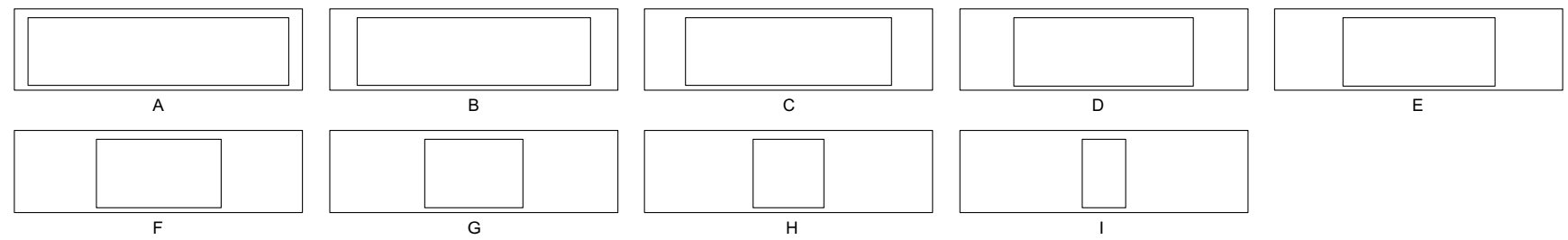

Figure 4. Resulting configurations for design variable: window width; change by: $1 \mathrm{~m}$ 


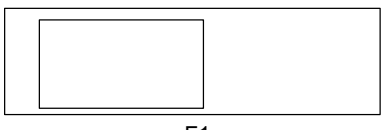

$\mathrm{F}$

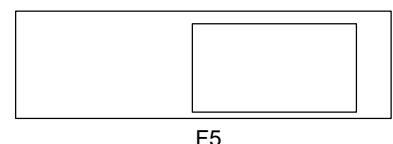

Figure 5. Resulting configurations for design variable: window location; change by: $1 \mathrm{~m}$

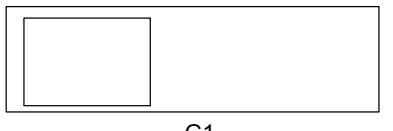

G1

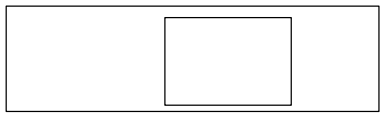

G5

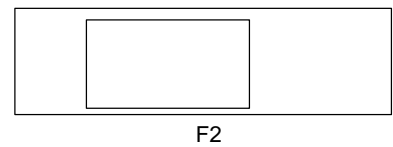

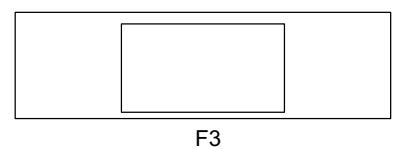

F3

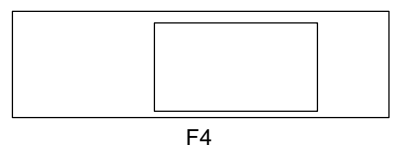

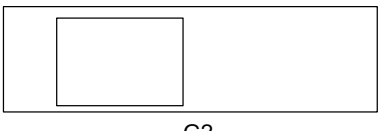

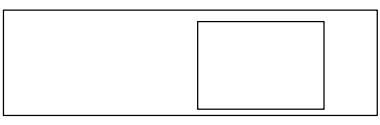

G6

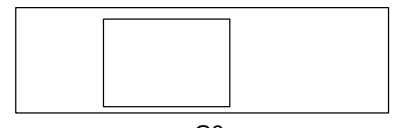

G3

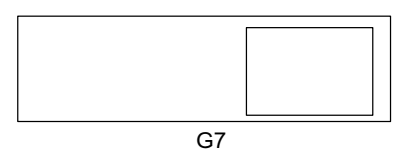

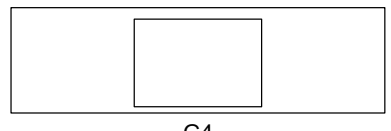

G4

Figure 6. Resulting configurations for design variable: window location; change by: $1 \mathrm{~m}$

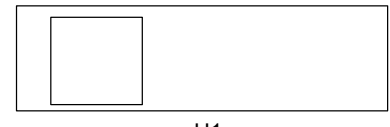

$\mathrm{H} 1$

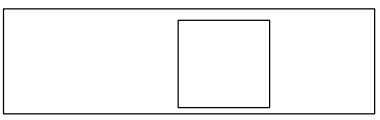

H5

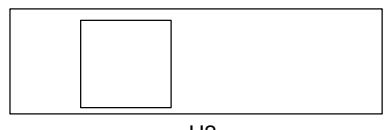

$\mathrm{H} 2$

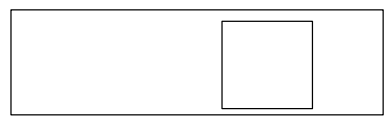

$\mathrm{H} 6$

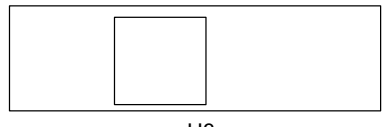

H3

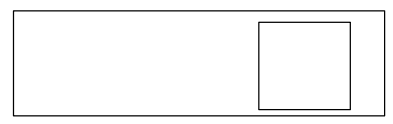

$\mathrm{H} 7$

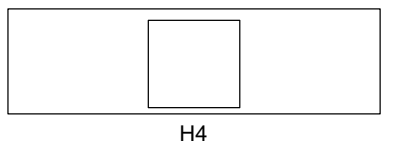

$\mathrm{H} 4$

Figure 7. Resulting configurations for design variable: window location; change by: $1 \mathrm{~m}$

\subsection{Neural Network Training Performance}

So as to determine a suitable network architecture for each prediction problem, the training performance was measured for networks with three to 25 neurons in the hidden layer. The Mean Squared Error (MSE) was used to measure training performance. ANN training was stopped when either the lowest possible MSE was reached at 1000 training Epochs or when the MSE worsened six consecutive times on the validation set. As aforementioned, each network architecture was trained ten times and the architecture with the lowest MSE in ten runs was recalled for DA predictions.

DA prediction results were generated in three ways: using the network with the lowest MSE, using the average output of the five networks with the lowest MSE for that architecture and by averaging the output of all ten trained networks. This was done to improve generalization [22]. Prediction accuracies and effectiveness of this are compared in the results section.

\subsection{Measurements of Prediction Accuracy}

The neural network output (between -1 and 1) was scaled back to DA levels before assessing the prediction accuracy. The accuracy measures used in this study are the Mean Absolute Error (MAE), the Root Mean Squared Error
(RMSE) and the Mean Biased Error (MBE). MAE, RMSE and $\mathrm{MBE}$ are calculated by using the following equations where $\mathrm{P}$ is the predicted, and $\mathrm{T}$ the simulated DA value:

$$
\begin{aligned}
M A E & =\frac{\sum_{t=1}^{n}\left|P_{t}-T_{t}\right|}{n} \\
R M S E & =\sqrt{\frac{\sum_{t=1}^{n}\left(P_{t}-T_{t}\right)^{2}}{n}} \\
M B E & =\frac{\sum_{t=1}^{n}\left(P_{t}-T_{t}\right)}{n}
\end{aligned}
$$

Although both MAE and RMSE take large errors into account, RMSE places penalty on large errors and weighs them more heavily. A major difference between MAE and RMSE could therefore indicate either a small number of largely incorrect predictions or a larger number of moderately incorrect predictions [23]. The MBE was used to assess whether the trained architectures typically over or underestimate DA predictions and to gauge how far off the daylight evaluation of the entire space fell from the simulation. 


\section{RESULTS}

4.1 Network Architectures and Prediction Accuracies

For each training set, the number of hidden neurons was optimised with the custom script. The number of neurons that resulted in the lowest MSE are listed in the table alongside MAE, MBE and RMSE for each prediction.

\section{Neural Network Validation}

Predictions for $10 \%$ of randomly selected data from ' $\mathrm{A}$ ' were less than $1 \mathrm{DA}$ away from the simulated DA level (Table 1). This was the case for both the unshaded and shaded scenario. The RMSE yielded results close to the MAE, showing that there were hardly any outliers. The high accuracy showed that the network was correctly trained on the data and could be used for annual DA predictions. Additionally, MBA of less than 0.1 DA suggests that prediction give overall high accuracy on the evaluation of daylight without a noticeable tendency to either over-or underestimate daylight predictions. The neural network architecture that was best able to match training and target data and return a lowest MSE had 13 neurons in the hidden Layer.

\section{Predicting DA for Variations in Window Dimension}

The accuracy of predictions for $\mathrm{B}$ and $\mathrm{H}$ are listed in Table 2. The ANN model with 23 and 24 neurons in the hidden layer returned the lowest MSE. Predictions for B are below 3 DA away from the simulated results and estimates for both the shaded and unshaded scenario show similarly good results. Prediction results for $\mathrm{H}$ have a slightly lower accuracy: although the absolute error again is less than 3 DA, there is one outlier for the result: The MAE rises to 9.7 and $5 \mathrm{DA}$ when using the best trained network for predictions, indicating the neural network may have been over trained on the specific training and target data, making the network less suitable for generalizing or predicting for new cases [24]. Another possibility is that noise in the data may have been generated from the behaviour of the simulation tool itself. Diva works on the basis of threephase method and depending on the location and distance of sensor points to one another, results may be more or less representative of the grid in which the sensor point is located [25].

In general, over and underestimations cancelled each other out, meaning the overall estimation of daylight within the space would range around the same DA levels as the simulation. This was the case with one exception, whereby the best performing network underestimated predictions of $\mathrm{H}$ (obstructed) by a DA of $5 \%$. Outliers seemed to be prevalent in both cases for which the network performed less accurate, based on the RMSE for both.

Predicting DA for Variations in Window Location

Predicting DA for variation in window location yielded very similar results to the prior case. All predictions had a MAE of max. 3 DA from the actual result with one exceptional case of 7 MAE when using the network with the lowest MSE for predictions in which case the network largely over predicted DA for that scenario. Not considering this case, accuracies were not consistently better or worse for the unshaded and shaded scenario. Despite an additional input feature used for training, the best performing network architectures had either the same number of hidden neurons or were close in range to the prior case for changes in window dimension.

\section{Predicting DA for Variations in Window Dimension and Location}

Predictions on four completely new cases for variations in both window dimension and window location yielded slightly worse results as the mean absolute prediction error went up from 3 to around 4 DA. This difference however is marginal in terms of interpretation of the overall daylight performance. Over- and underestimations were slightly more pronounced on two of the cases (G2 and G7), suggesting that the magnitude of variation may be too great and additional training data from a more similar case may improve results. One ANN model was optimized for all four predictions and the architecture with 24 hidden neurons returned the best training performance.

\subsection{Best Performing Networks and their Ability to Generalize}

The greatest consistency in prediction results was obtained by averaging the output of all ten trained networks. Networks with the lowest MSE achieved the best as well as the worst prediction accuracies. Overall results largely fell in the same range of error between 1 and 3\% DA.

The number of hidden neurons that were best able to match input data and corresponding targets fell in the same range, with 21 to 24 neurons respectively. If, depending on the training data, completely different number of neurons had led to optimum results, this would have meant that multiple neural networks need to be trained depending on the design variables and the data sets. Instead, the observed result suggests consistency in the way that the neural nets learn and that convergence within the 'black box' may not be at random.

\subsection{Computation Time}

Daylight simulations on Diva for Rhino took between 10 and 15 minutes per case on a $2.6 \mathrm{GHz}$ Intel Core i7. The time required for training each ANN architecture took less than two minutes when provided with 726 data points. The training and optimization of ANNs used for predicting DA for variations in window dimension and location $(\mathrm{G})$ took slightly longer with 2 minutes and 15 seconds as the networks were provided with a dataset of 2057 training samples. Once a neural network architecture was trained, the recall for the DA predictions itself took less than 1 second. 


\begin{tabular}{|c|c|c|c|c|c|c|}
\hline \multicolumn{2}{|c|}{ Predicted case } & \multirow{2}{*}{$\begin{array}{c}\begin{array}{c}\text { No. of } \\
\text { hidden } \\
\text { neurons } \\
\text { with lowest } \\
\text { MSE }\end{array} \\
13 \\
\end{array}$} & \multirow{2}{*}{$\begin{array}{c}\begin{array}{c}\text { Errors of } \\
\text { best trained } \\
\text { ANN }\end{array} \\
0.51 \\
-0.06 \\
0.63\end{array}$} & \multirow{2}{*}{$\begin{array}{c}\begin{array}{c}\text { Errors of } \\
\text { top } 5 \\
\text { trained } \\
\text { ANNs }\end{array} \\
0.41 \\
-0.10 \\
0.49\end{array}$} & \multirow{2}{*}{$\begin{array}{c}\begin{array}{c}\text { Errors of } 10 \\
\text { trained } \\
\text { ANNs }\end{array} \\
0.48 \\
-0.07 \\
0.55\end{array}$} & \multirow{2}{*}{$\begin{array}{c}\begin{array}{c}\text { Elapsed } \\
\text { time in } \\
\text { seconds }\end{array} \\
45.66\end{array}$} \\
\hline $\begin{array}{c}\text { A - } \\
\text { Unshaded }\end{array}$ & $\begin{array}{c}\text { MAE } \\
\text { MBE } \\
\text { RMSE }\end{array}$ & & & & & \\
\hline $\begin{array}{c}\text { A - } \\
\text { Shaded }\end{array}$ & $\begin{array}{c}\text { MAE } \\
\text { MBE } \\
\text { RMSE }\end{array}$ & 13 & $\begin{array}{l}0.55 \\
0.09 \\
0.65\end{array}$ & $\begin{array}{c}0.51 \\
-0.05 \\
0.65\end{array}$ & $\begin{array}{c}0.54 \\
-0.02 \\
0.72\end{array}$ & 40.31 \\
\hline
\end{tabular}

Table 1. Prediction accuracy: Neural Network validation

\begin{tabular}{|c|c|c|c|c|c|c|}
\hline Predic & case & $\begin{array}{c}\text { No. of } \\
\text { hidden } \\
\text { neurons }\end{array}$ & $\begin{array}{c}\text { Errors of } \\
\text { best trained }\end{array}$ & $\begin{array}{l}\text { Errors of } \\
\text { top } 5\end{array}$ & $\begin{array}{c}\text { Errors of } 10 \\
\text { trained }\end{array}$ & $\begin{array}{c}\text { Elapsed } \\
\text { time in }\end{array}$ \\
\hline $\begin{array}{c}\text { B - } \\
\text { Unshaded }\end{array}$ & $\begin{array}{c}\text { MAE } \\
\text { MBE } \\
\text { RMSE }\end{array}$ & 23 & $\begin{array}{l}1.01 \\
0.92 \\
1.22\end{array}$ & $\begin{array}{l}2.55 \\
2.67 \\
3.30\end{array}$ & $\begin{array}{l}1.67 \\
1.55 \\
2.09\end{array}$ & 76.71 \\
\hline $\begin{array}{c}\text { H - } \\
\text { Unshaded }\end{array}$ & $\begin{array}{c}\text { MAE } \\
\text { MBE } \\
\text { RMSE }\end{array}$ & 23 & $\begin{array}{c}9.77 \\
0.28 \\
13.71\end{array}$ & $\begin{array}{c}2.44 \\
-1.29 \\
3.16\end{array}$ & $\begin{array}{c}1.60 \\
-1.13 \\
2.16\end{array}$ & 82.47 \\
\hline $\begin{array}{c}\text { B - } \\
\text { Shaded }\end{array}$ & $\begin{array}{c}\text { MAE } \\
\text { MBE } \\
\text { RMSE }\end{array}$ & 24 & $\begin{array}{l}1.48 \\
0.56 \\
1.81\end{array}$ & $\begin{array}{l}1.04 \\
0.13 \\
1.33\end{array}$ & $\begin{array}{l}0.96 \\
0.36 \\
1.18\end{array}$ & 75.45 \\
\hline $\begin{array}{c}\text { H - } \\
\text { Shaded }\end{array}$ & $\begin{array}{c}\text { MAE } \\
\text { MBE } \\
\text { RMSE }\end{array}$ & 23 & $\begin{array}{c}5.04 \\
-4.97 \\
7.11\end{array}$ & $\begin{array}{c}2.83 \\
-2.34 \\
4.15\end{array}$ & $\begin{array}{c}2.74 \\
-1.86 \\
3.85\end{array}$ & 92.70 \\
\hline
\end{tabular}

Table 2. Prediction accuracy: Variations in window dimension

\begin{tabular}{|c|c|c|c|c|c|c|}
\hline Predic & case & $\begin{array}{c}\text { No. of } \\
\text { hidden } \\
\text { neurons }\end{array}$ & $\begin{array}{c}\text { Errors of } \\
\text { best trained }\end{array}$ & $\begin{array}{c}\text { Errors of } \\
\text { top } 5 \\
\text { trained }\end{array}$ & $\begin{array}{c}\text { Errors of } 10 \\
\text { trained }\end{array}$ & $\begin{array}{c}\text { Elapsed } \\
\text { time in }\end{array}$ \\
\hline $\begin{array}{c}\text { H2 - } \\
\text { Unshaded }\end{array}$ & $\begin{array}{c}\text { MAE } \\
\text { MBE } \\
\text { RMSE }\end{array}$ & 24 & $\begin{array}{l}2.39 \\
1.92 \\
3.68\end{array}$ & $\begin{array}{l}1.64 \\
0.56 \\
2.32\end{array}$ & $\begin{array}{c}2.30 \\
-1.94 \\
2.94\end{array}$ & 77.17 \\
\hline $\begin{array}{c}\text { H6 - } \\
\text { Unshaded }\end{array}$ & $\begin{array}{c}\text { MAE } \\
\text { MBE } \\
\text { RMSE }\end{array}$ & 23 & $\begin{array}{l}7.73 \\
6.84 \\
9.90\end{array}$ & $\begin{array}{l}2.21 \\
1.78 \\
3.00\end{array}$ & $\begin{array}{l}1.40 \\
0.87 \\
1.95\end{array}$ & 68.41 \\
\hline $\begin{array}{c}\text { H2 - } \\
\text { Shaded }\end{array}$ & $\begin{array}{c}\text { MAE } \\
\text { MBE } \\
\text { RMSE }\end{array}$ & 23 & $\begin{array}{c}2.06 \\
-1.03 \\
3.55\end{array}$ & $\begin{array}{l}3.58 \\
3.46 \\
5.38\end{array}$ & $\begin{array}{l}2.15 \\
1.68 \\
3.14\end{array}$ & 61.28 \\
\hline $\begin{array}{c}\text { H6 - } \\
\text { Shaded }\end{array}$ & $\begin{array}{c}\text { MAE } \\
\text { MBE } \\
\text { RMSE }\end{array}$ & 21 & $\begin{array}{c}2.25 \\
-0.60 \\
3.68\end{array}$ & $\begin{array}{c}3.48 \\
-0.15 \\
4.59\end{array}$ & $\begin{array}{c}3.46 \\
-2.41 \\
4.68\end{array}$ & 66.51 \\
\hline
\end{tabular}

Table 3. Prediction accuracy: Variations in window location 


\begin{tabular}{|c|c|c|c|c|c|c|}
\hline \multicolumn{2}{|c|}{ Predicted case } & \multirow{2}{*}{$\begin{array}{c}\text { No. of } \\
\text { hidden } \\
\text { neurons } \\
\text { with lowest } \\
\text { MSE }\end{array}$} & \multirow{2}{*}{$\begin{array}{c}\begin{array}{c}\text { Errors of } \\
\text { best trained } \\
\text { ANN }\end{array} \\
3.99 \\
0.15 \\
6.96\end{array}$} & \multirow{2}{*}{$\begin{array}{c}\begin{array}{c}\text { Errors of } \\
\text { top 5 } \\
\text { trained } \\
\text { ANNs }\end{array} \\
2.47 \\
0.95 \\
3.98\end{array}$} & \multirow{2}{*}{$\begin{array}{c}\begin{array}{c}\text { Errors of } \mathbf{1 0} \\
\text { trained } \\
\text { ANNs }\end{array} \\
2,29 \\
-1,33 \\
4.20\end{array}$} & \multirow{5}{*}{$\begin{array}{c}\text { Elapsed } \\
\text { time in } \\
\text { seconds }\end{array}$} \\
\hline $\begin{array}{c}\text { G1 - } \\
\text { Shaded }\end{array}$ & $\begin{array}{c}\text { MAE } \\
\text { MBE } \\
\text { RMSE }\end{array}$ & & & & & \\
\hline $\begin{array}{c}\text { G2 - } \\
\text { Shaded }\end{array}$ & $\begin{array}{c}\text { MAE } \\
\text { MBE } \\
\text { RMSE }\end{array}$ & \multirow{3}{*}{24} & $\begin{array}{l}3.47 \\
3.37 \\
5.07\end{array}$ & $\begin{array}{l}4.51 \\
4.46 \\
6.39\end{array}$ & $\begin{array}{l}3,78 \\
3.68 \\
5.48\end{array}$ & \\
\hline $\begin{array}{c}\text { G6 - } \\
\text { Shaded }\end{array}$ & $\begin{array}{c}\text { MAE } \\
\text { MBE } \\
\text { RMSE }\end{array}$ & & $\begin{array}{c}2.71 \\
-2.52 \\
3.44\end{array}$ & $\begin{array}{c}2.90 \\
-2.59 \\
3.76\end{array}$ & $\begin{array}{c}1,39 \\
-0.98 \\
2.02\end{array}$ & \\
\hline $\begin{array}{c}\text { G7 - } \\
\text { Shaded }\end{array}$ & $\begin{array}{c}\text { MAE } \\
\text { MBE } \\
\text { RMSE }\end{array}$ & & $\begin{array}{c}3.46 \\
-4.31 \\
6.11\end{array}$ & $\begin{array}{l}3.32 \\
4.22 \\
5.38\end{array}$ & $\begin{array}{l}2,38 \\
2.12 \\
3.22\end{array}$ & \\
\hline
\end{tabular}

Table 4. Prediction accuracy: Variations in window dimension and location

\section{CONCLUSION AND RECOMMENDATIONS FOR FUTURE RESEARCH}

It should be noted that this study investigates the potential of ANNs to estimate DA levels but does not infer on their ability to fully re-establish the DA measures that results from backward ray-tracing to the sky patches and the respective climate-data, which would require further research with more detailed data. Instead, observations are made on how close ANN predictions get to simulated DA levels as well as the consistency of predictions. This was done to gauge the suitability of ANNs to partially replace time-consuming simulations in daylight performance-based design explorations. Predictions typically fell within 3\% DA on average away from simulated DA level of each sensor point and were more accurate in terms of evaluating daylight performance of the entire space, as indicated by the MBE results. A typical MBE close to 0 showed that there was no particular tendency to over or underestimate daylight levels.

One of the main limitations pertains to the fact that the design variables need to be determined before training the ANN. This would make ANNs more suitable for

\section{REFERENCES}

[1] C. Maesano and I. Annesi-Maesano, Impact of Lighting on School Performance in European Classrooms, Paris, 2012.

[2] J. A. Veitch, J. Christoffersen, and A. D. Galasiu, Daylight and View through Residential Windows: Effects on Well-being, Residential daylighting and Well-being, p.1-6, 2013.

[3] C. F. Reinhart, J. Mardaljevic, and Z. Rogers, Dynamic Daylight Performance Metrics for application in design optimisation problems. In terms of robustness of the ANN models, outliers in prediction accuracy were repeatedly found when employing the models with the best training performance, possibly suggesting an overfitting of the networks to the training data. The results of this study show that training multiple networks and averaging their output provides more consistent prediction accuracies.

Although we were able to predict daylight levels for changes in façade design in terms of window dimension and location, further research is needed to test prediction accuracy for more complex façade changes. Improvements in input feature selection and ANN settings such as a slower convergence rate are expected to improve results. The sample size used for training and the selection of data used as the validation set for early stopping are further factors that influence the results. A more in-depth exploration is still needed to validate the method and future work will investigate the adaptability of ANNs to predict DA levels under changing design with multiple design parameters.

Sustainable Building Design, J. Illum. Eng. Soc. North Am., vol. 3, no. 1, pp. 7-31, 2013.

[4] C. F. Reinhart and O. Walkenhorst, Validation of dynamic RADIANCE-based daylight simulations for a test office with external blinds, Energy Build., vol. 33, no. 7, pp. 683-697, 2001.

[5] J. Hu and S. Olbina, Simulation-Based Model for Integrated Daylighting System Design, J. Comput. Civ. Eng., vol. 28, 2014.

[6] T. Kazanasmaz, M. Gunaydin, and S. Binol, Artificial neural networks to predict daylight 
illuminance in office buildings, Building and Environment, vol. 44, pp. 1751-1757, 2009.

[7] P. Sajda, Neural Networks, in Encyclopedia of the Human Brain Volume 3, V. S. Ramachandran, Ed. Columbia University, USA: Academic Press, 2002, pp. 373-383.

[8] S. Pattanasethanon, C. Lertsatitthanakorn, and S. Atthajariyakul, An accuracy assessment of an empirical sine model, a novel sine model and an artificial neural network model for forecasting illuminance / irradiance on horizontal plane of all sky types at Mahasarakham, Thailand, vol. 49, pp. 1999-2005, 2008.

[9] S. Zhou and D. Liu, Prediction of Daylighting and Energy Performance Using Artificial Neural Network and Support Vector Machine, Am. J. Civ. Eng. Archit. Vol. 3, 2015, Pages 1-8, vol. 3, no. 3A, pp. 1-8, 2015.

[10] J. Hu and S. Olbina, Illuminance-based slat angle selection model for automated control of split blinds, Build. Environ., vol. 46, no. 3, pp. 786-796, 2011.

[11] S. Janjai and P. Plaon, Estimation of sky luminance in the tropics using artificial neural networks: Modeling and performance comparison with the CIE model, Appl. Energy, vol. 88, no. 3, pp. 840$847,2011$.

[12] C.-L. Lorenz and W. Jabi, Predicting Daylight Autonomy Metrics Using Machine Learning, in Int. Conf.e for Sustainable Design of the Built Environment, 2017.

[13] F. Burden and D. Winkler, Artificial Neural Networks: Methods and Applications. Humana Press, 2009.

[14] R. Hecht-Nielsen, Theory of the Backpropagation Neural Network, in Proc. of the Int. Joint IEEE Conf.e on Neural Networks, 1989, pp. 593-605.

[15] Y. LeCun, L. Bottou, G. B. Orr, and K.-R. Müller, Efficient BackProp, in Neural Networks: Tricks of the trade, Berlin, Germany: Springer, 1998.

[16] D. Marquardt, An Algorithm for Least-Squares Estimation of Nonlinear Parameters, SIAM J. Appl. Math., vol. 11, no. 2, pp. 431-441, 1963.

[17] B. M. Wilamowski, Neural network architectures and learning algorithms, IEEE Ind. Electron. Mag., vol. 3, no. 4, pp. 56-63, 2009.

[18] C. Joe, Build Neural Network With MS Excel. 2009.

[19] J. Conraud-bianchi, A Methodology for the Optimization of Building Energy, Thermal, and Visual Performance. 2008.

[20] M. Kayri, Predictive Abilities of Bayesian Regularization and Levenberg-Marquardt Algorithms in Artificial Neural Networks: A Comparative Empirical Study on Social Data," Math. Comput. Appl., vol. 21, no. 2, p. 20, 2016.

[21] A. McNeil and E. S. Lee, A validation of the Radiance three-phase simulation method for modelling annual daylight performance of optically complex fenestration systems," J. Build. Perform. Simul., vol. 6, no. 1, pp. 24-37, 2012.

[22] The MathWorks Inc., Improve Neural Network Generalization and Avoid Overfitting, R2017b Documentation.

https://uk.mathworks.com/help/nnet/ug/improveneural-network-generalization-and-avoidoverfitting.html\#bss4gz0-35. As of 20 Aug 2017.

[23] J. M. Twomey and A. E. Smith, Performance measures, consistency, and power for artificial neural network models, Math. Comput. Model., vol. 21, no. 1/2, pp. 243-258, 1995.

[24] M. Buckland, AI Techniques for Game Programming. Cincinnati, Ohio: Premier Press, 2002.

[25] E. Brembilla and J. Mardaljevic, Climate-Based Daylight Modelling: The What, the Why and the How, CIBSE Daylight Group, 2016. 